\title{
Circadian Blood Pressure Variations in Postmenopausal Females with Hypertension
}

\author{
Dan Su, ${ }^{1} \mathrm{MD}$, Anqi Song, ${ }^{1} \mathrm{MD}$, Bin Yan, ${ }^{2} \mathrm{MD}$, Qi Guo, ${ }^{1} \mathrm{MD}$, Ya Gao, ${ }^{3} \mathrm{MD}$, Yanhua Zhou, ${ }^{4} \mathrm{MD}$, \\ Fuling Zhou, ${ }^{5} \mathrm{MD}$, Guang Yang, ${ }^{6} \mathrm{MD}$ and Chaoying Zhang, ${ }^{1} \mathrm{MD}$
}

\begin{abstract}
Summary
The abnormalities of blood pressure (BP) nocturnal decline have been found to be predictive for carotid plaque and lacunar infarction in patients with hypertension. In this study, BP dipping patterns in postmenopausal females with hypertension were investigated. The nocturnal decline of systolic BP (SBP) was evaluated using 24-hour ambulatory BP monitoring (ABPM). A total of 163 postmenopausal females were eventually included in our study. The prevalence of reverse-dipper BP pattern was $32.3 \%$ in females with menopause age in their $40 \mathrm{~s}$ and $40 \%$ in their 50s. However, after multivariate logistic regression analysis, menopause age was shown to be an independent risk factor for BP reverse dipping (Odds ratio [OR] 1.148; 95\%CI 1.020 - $1.292 ; P$ $=0.020)$. Moreover, menopause age was negatively correlated with the decline rate of nocturnal SBP ( $r=$ -0.159; $P<0.05)$ and diastolic BP $(r=-0.161 ; P<0.05)$. Our study suggested that the menopause age might serve as a risk factor for reverse-dipper BP pattern in postmenopausal females with hypertension.
\end{abstract}

Key words: Essential hypertension, Circadian BP patterns, Menopause age

(Int Heart J 2018; 59: 361-366)

Y oung women are less likely to suffer from cardiovascular disease (CVD) than men, although this advantage is rapidly reversed after menopause, which is considered because of the protective effect of estrogen on the cardiovascular system. ${ }^{1}$ CVD rates in women after menopause are 2 - 3 times those of the same age before menopause. ${ }^{2)}$ Furthermore, studies have indicated a higher risk of cardiovascular heart disease (CHD), heart failure (HF), CVD mortality and overall mortality in women who experience premature or early-onset menopause. ${ }^{3,4)}$ The prevalence of hypertension, which is one of the main risk factors for CVD, is known to increase with age, ${ }^{5)}$ so proper management of blood pressure (BP) in peri- and postmenopausal women is essential for the prevention of CVD in later life.

Ambulatory blood pressure monitoring (ABPM), which offers us the average BP, variability, and circadian dipping status across a 24-hour (24-h) period, has better reproducibility and prognostic superiority compared with standard clinical measurements. ${ }^{6-9)}$ According to the ratio of systolic blood pressure (SBP) at night and day based on ABPM data, circadian BP variations were used to be divided into dipper (mean nocturnal SBP drops $10 \mathrm{mmHg}$ or more than that in daytime) and non-dipper (all other subjects). As a particular variant of non-dipper pattern, reverse-dipper defined as an increase in SBP at night, which was found to be associated with higher incidence of cardiovascular events. ${ }^{10)}$ Consistently, our previous study also suggested that BP reverse dipping was the real risk factor for carotid atherosclerosis, diabetes mellitus (DM) and lacuna infarction. ${ }^{11-13)}$

We conducted this study to investigate the relationship between menopause age and reverse-dipper BP pattern. Moreover, we tried to evaluate the potential association of menopause age with the decline rate of nocturnal $\mathrm{BP}$ in all hypertensive patients.

\section{Methods}

Study population: A total of 163 postmenopausal females with essential hypertension were eventually included in our study. All inpatients were recruited from January 2012 to June 2014 in Xi'an, Shaanxi Province, China. Data were extracted from our entire in-patient ABPM service database. Age at menopause was defined as the age at last menstrual period or bilateral oophorectomy. Hypertension patients was defined as SBP $\geq 140$ $\mathrm{mmHg}$ and/or DBP $\geq 90 \mathrm{~mm} \mathrm{Hg}$ in casual office record-

From the ${ }^{1}$ Department of Cardiology, the Second Affiliated Hospital, Xi' an Jiaotong University, Xi' an, China, ${ }^{2}$ Department of Clinical Research Center, the First Affiliated Hospital, Xi'an Jiaotong University, Xi'an, China, ${ }^{3}$ Department of Emergency Medicine, the Second Affiliated Hospital, Xi'an Jiaotong University, Xi'an, China, ${ }^{4}$ Department of Cardiology, Xianyang People's Hospital, Xi' an, China, '5epartment of Hematology, Zhongnan Hospital, Wuhan University, Wuhan, China and ${ }^{6}$ Department of Cardiology, Shaanxi Provincial People's Hospital, Xi'an, China.

Address for correspondence: Chaoying Zhang, MD, Department of Cardiology, The Second Affiliated Hospital, Xi'an Jiaotong University, 157 Xi5 Lu, Xi'an, Shaanxi 710004, China. E-mail: cyzhang@mail.xjtu.edu.cn or Guang Yang, MD, Department of Cardiology, Shaanxi Provincial People's Hospital, 256 Friendship Road, Xi'an, Shaanxi 710068, China. E-mail: zjwcoconut@126.com

Received for publication April 9, 2017. Revised and accepted May 27, 2017.

Released in advance online on J-STAGE February 23, 2018.

doi: 10.1536/ihj.17-206

All rights reserved by the International Heart Journal Association. 
Table I. Characteristics of the Study Population by Dipping Status

\begin{tabular}{lcccc}
\hline \multicolumn{1}{c}{ Variable } & Dippers & Non-dippers & Reverse-dippers & $P$ \\
\hline Patients, $n$ & 18 & 87 & 58 & \\
Age, years & $64.4 \pm 9.6$ & $64.5 \pm 10.3$ & $64.6 \pm 9.1$ & 0.998 \\
Diabetes, $n(\%)$ & $3(16.7)$ & $26(29.9)$ & $20(34.5)$ & 0.325 \\
History of smoking, $n(\%)$ & $0(0)$ & $0(0)$ & $0(0)$ & -- \\
Menopausal age, years & $45.8 \pm 6.0$ & $48.1 \pm 4.0^{*}$ & $48.6 \pm 3.6^{\#}$ & 0.054 \\
Triglycerides $(\mathrm{mmol} / \mathrm{L})$ & $1.9 \pm 0.8$ & $1.8 \pm 1.0$ & $1.7 \pm 1.3$ & 0.728 \\
Total cholesterol $(\mathrm{mmol} / \mathrm{L})$ & $5.0 \pm 0.8$ & $4.7 \pm 0.9$ & $4.9 \pm 1.1$ & 0.391 \\
$\quad$ HDL-C $(\mathrm{mmol} / \mathrm{L})$ & $1.3 \pm 0.2$ & $1.3 \pm 0.3$ & $1.3 \pm 0.3$ & 0.405 \\
LDL-C $(\mathrm{mmol} / \mathrm{L})$ & $3.0 \pm 0.7$ & $2.7 \pm 0.7$ & $2.9 \pm 0.9$ & 0.260 \\
VLD-C $(\mathrm{mmol} / \mathrm{L})$ & $0.6 \pm 0.4$ & $0.7 \pm 0.5$ & $0.7 \pm 0.6$ & 0.918 \\
24 h-SBP, ABPM $(\mathrm{mmHg})$ & $132.1 \pm 12.1$ & $133.7 \pm 14.2$ & $133.5 \pm 15.3$ & 0.911 \\
24 h-DBP, ABPM (mmHg) & $75.7 \pm 9.2$ & $73.7 \pm 8.7$ & $75.9 \pm 8.6$ & 0.286 \\
SBP-awakening, $(\mathrm{mmHg})$ & $136.2 \pm 12.9$ & $134.7 \pm 14.2$ & $131.9 \pm 14.2$ & 0.384 \\
DBP-awakening, $(\mathrm{mmHg})$ & $78.7 \pm 9.8$ & $74.2 \pm 7.9$ & $75.6 \pm 8.2$ & 0.100 \\
SBP-bedtime, $(\mathrm{mmHg})$ & $116.6 \pm 11.0$ & $128.3 \pm 13.8^{*}$ & $137.0 \pm 14.5^{* \#}$ & 0.001 \\
DBP-bedtime, $(\mathrm{mmHg})$ & $64.3 \pm 8.2$ & $69.8 \pm 8.4 *$ & $76.2 \pm 9.2^{* \#}$ & 0.001 \\
\hline P & &
\end{tabular}

$P$ for difference among the 3 groups. ABPM indicates ambulatory blood pressure monitoring; DBP, diastolic blood pressure; HDL-C, high-density lipoprotein cholesterol; LDL-C, low-density lipoprotein cholesterol; SBP, systolic blood pressure; and VLD-C, very low-density lipoprotein cholesterol. *Control with dipper group, $P<0.05$, \#Control with non-dipper group, $P<$ 0.05 .

ing, or daytime (or awake) $\mathrm{SBP} \geq 135 \mathrm{mmHg}$ and/or DBP $\geq 85 \mathrm{~mm} \mathrm{Hg}$, or night-time (or asleep) $\mathrm{SBP} \geq 120 \mathrm{~mm}$ $\mathrm{Hg}$ and/or DBP $\geq 70 \mathrm{mmHg}$ in ABPM. DM was defined as a fasting blood glucose level $\geq 7.0 \mathrm{mmol} / \mathrm{L}$, non-fasting blood glucose $\geq 11.1 \mathrm{mmol} / \mathrm{L}$, a self-reported physician diagnosis of DM, or current use of antidiabetic medication. The nocturnal decline of SBP was evaluated using 24-hour ABPM. In this study, the circadian decline rate of SBP fluctuating from $10 \%$ to $20 \%$ was considered as BP dipping pattern. Non-dipper ( $0 \%$ to $10 \%$ SBP fall) and reverse-dipper (SBP nocturnal rise) were also investigated. In addition, no extreme dipper ( $>20 \%$ nocturnal SBP fall) was observed in this study.

The hypertensive patients were excluded if they 1) were $<30$ or $>90$ years old; 2) were under antihypertensive treatment; 3) had a BP over $160 / 100 \mathrm{mmHg}$; 4) were night workers; 5) had acute stroke or myocardial infarction within the past 6 months; 6) had sleep apnea syndrome; 7) were diagnosed with secondary hypertension; 8) could not tolerate the ABPM; 9) had other chronic diseases.

ABPM assessment: All patients underwent 24-hour ABPM using an oscillometric device (Spacelabs 90207; Spacelabs, Redmond, WA). BP was recorded at every 15 minutes from 7:00AM to 11:00PM (diurnal BP values) and every 30 minutes from 11:00PM to 7:00AM (nocturnal BP values). Subjects were instructed not to exercise before or during the monitoring period and to pause momentarily and maintain their body position during each BP measurement. Monitoring was done on a working day. Patients were asked to attend their usual activities but to keep motionless at the time of measurement. Only recordings of good technical quality ( $>70 \%$ of valid BP measurements) were included in final analyses. ${ }^{8}$ All ABPM recordings were manually edited by two individual physicians unaware of the objective and risk factors. Values of $\mathrm{SBP}<70$ or $>250 \mathrm{mmHg}, \mathrm{DBP}<40$ or $>150 \mathrm{mmHg}$, and heart rate $<40$ or $>150$ beats per minute were excluded from the recording. Fewer than $3 \%$ of the BP readings were rejected as artifacts on the basis of these criteria. The percent change in nocturnal blood pressure decline was calculated as follows: (mean diurnal BP mean nocturnal $\mathrm{BP}) /$ mean diurnal $\mathrm{BP} \times 100$.

Laboratory tests: Baseline demographic, clinical and laboratory data were carefully recorded. Peripheral blood samples were drawn routinely from patients in a fasting state. Plasma concentrations of glucose, lipids and other biochemical parameters were determined at the Biochemistry Department in our hospital.

Statistical analysis: Descriptive statistics are presented as percentages for discrete variables and mean \pm SD for continuous normally distributed variables. An ANOVA test was used for statistical comparison of data that were normal distributed. The distribution of three circadian BP patterns among postmenopausal females with different menopause age was analyzed using a chi-squared test. Multivariate logistic regression analyses were applied to explore the relationship between the relevant variable and ABPM result. Bivariate correlation analysis was employed to examine the association between menopause age and the decline rate of nocturnal BP. $P$-values $<0.05$ were considered statistically significant for all analyses. All statistical analyses were performed using the SPSS software package version 18.0 (SPSS Inc., Chicago, IL, USA).

The study protocol was approved by the ethics committee of the Second Affiliated Hospital, Xi' an Jiaotong University. All subjects consented to participate after been informed of the nature and purpose of the study.

\section{Results}

Clinical and laboratory characteristics of the study population: The demographic and clinical characteristics of patients in different groups according to circadian vari- 


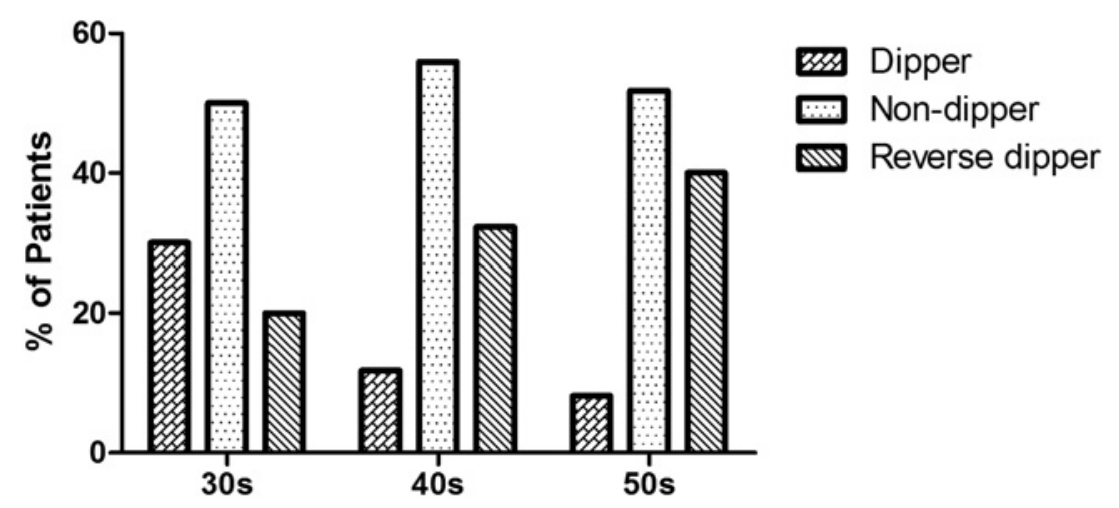

Figure 1. The distribution of three circadian BP patterns among postmenopausal females with different menopause age.

Table II. Univariate and Multivariate Logistic Regression Analysis for Circadian BP Pattern

\begin{tabular}{lllllll}
\hline \multirow{2}{*}{ Variable } & \multicolumn{2}{c}{ Reversed-dipper versus Dipper } & \multicolumn{2}{c}{ Non-dipper versus dipper } & \multicolumn{2}{c}{ Reversed-dipper versus Non-dipper } \\
& \multicolumn{1}{c}{ OR $(95 \% \mathrm{CI})$} & $P$ & OR $(95 \% \mathrm{CI})$ & $P$ & OR $(95 \% \mathrm{CI})$ & $P$ \\
\hline Age & $0.985(0.930-1.044)$ & 0.619 & $0.986(0.933-1.043)$ & 0.628 & $0.999(0.963-1.036)$ & 0.954 \\
Diabetes & $2.775(0.674-11.435)$ & 0.158 & $2.209(0.577-8.757)$ & 0.259 & $1.256(0.602-2.621)$ & 0.543 \\
Triglycerides & $0.799(0.457-1.396)$ & 0.430 & $0.983(0.591-1.635)$ & 0.947 & $0.813(0.559-1.181)$ & 0.277 \\
Total cholesterol & $1.024(0.554-1.893)$ & 0.94 & $0.779(0.430-1.411)$ & 0.410 & $1.315(0.911-1.899)$ & 0.140 \\
Menopausal age & $1.148(1.020-1.292)$ & 0.02 & $1.113(1.000-1.239)$ & 0.050 & $1.031(0.943-1.127)$ & 0.497 \\
\hline
\end{tabular}

Global $P$ for difference between the 3 groups. CI indicates confidence interval; and OR, odds ratio.

ations of BP were shown in Table I. A total of 163 hypertensive patients, including 18 dippers $(11.0 \%), 87$ nondippers $(53.4 \%)$ and 58 reverse-dippers $(35.6 \%)$, were involved in our study. There were no significant differences in age, diabetes, history of smoking, triglycerides, and cholesterol among the three groups. In addition, there was a significant difference $(P=0.016)$ in menopause age between reverse dippers $(48.6 \pm 3.6)$ and dippers $(45.8 \pm$ 6.0).

Association of menopause age with circadian BP patterns: The distribution of three circadian BP patterns (dipper, non-dipper, reverse-dipper) among postmenopausal females with different menopause age was analyzed using a chi-squared test. As shown in Figure 1, the prevalence of reverse-dipper BP pattern was $32.3 \%$ in females with menopause age of $40 \mathrm{~s}$ and $40 \%$ at 50 s.

Furthermore, a univariate multinomial logistic regression analysis using stepwise selection process was performed in our study. Our univariate models included age, diabetes, smoking, menopause age, triglycerides, HDL-C, LDL-C, VLD-C, 24h-SBP, 24h-DBP, SBP-awakening, SBP-bedtime, DBP-awakening and DBP-bedtime. The variables found to have significance and acceptable collinearity in univariate models were included in the multinomial analyses. It was discovered that menopause age was significantly different when comparing reversedipper BP pattern with dipper pattern (Table II). This model suggested that menopause age was an independent risk factor for BP reverse dipping with essential hypertension (Odds ratio [OR] 1.148; 95\% CI $1.020-1.292 ; P=$ 0.020).

Correlation between menopause age and decline rate of nocturnal BP: Nocturnal BP has been considered to be a more important risk factor than office BP since it is not affected by the "white coat effect."14,15) A decrease of 10\%$20 \%$ in night-time BP has been considered to be a normal level of dipping. Lack of nocturnal reduction that is less than $10 \%$ compared to the day time BP value has been associated with increased CVD risk factors. ${ }^{16)}$ In order to further clarify the relationship between menopause age and the decline of nocturnal BP, bivariate correlation analysis was performed. Consistently, we found that menopause age was negatively correlated with the decline rate of nocturnal SBP $(r=-0.159 ; P<0.05)$ and DBP $(r$ $=-0.161 ; P<0.05)$ (Figure 2).

\section{Discussion}

BP has a circadian rhythm in which it decreases at night and increases in the morning; nocturnal dipping of blood pressure is an important physiologic phenomenon. Absence of the normal nocturnal fall in SBP is associated with increased morbidity and mortality, and poses a substantial risk even after antihypertensive treatment. ${ }^{17-19)}$ Studies have reported the association of non-dipper pattern of BP with carotid intima-media thickness (CIMT), which was assessed by B-mode ultrasound and widely performed to evaluate the risk and prognosis of cardiovascular diseases. ${ }^{20,21)}$ Patients with a non-dipper pattern have more target-organ damage induced by hypertension, such as left ventricular hypertrophy, microalbuminuria, and reduced arterial compliance. Reverse-dipper hypertension, which was categorized as a variant of non-dipper, was recently regarded as an independent predictor for graft outcome and closely associated with cardiovascular injuries in chronic kidney disease. ${ }^{22,23)}$ In addition, reverse-dipper 

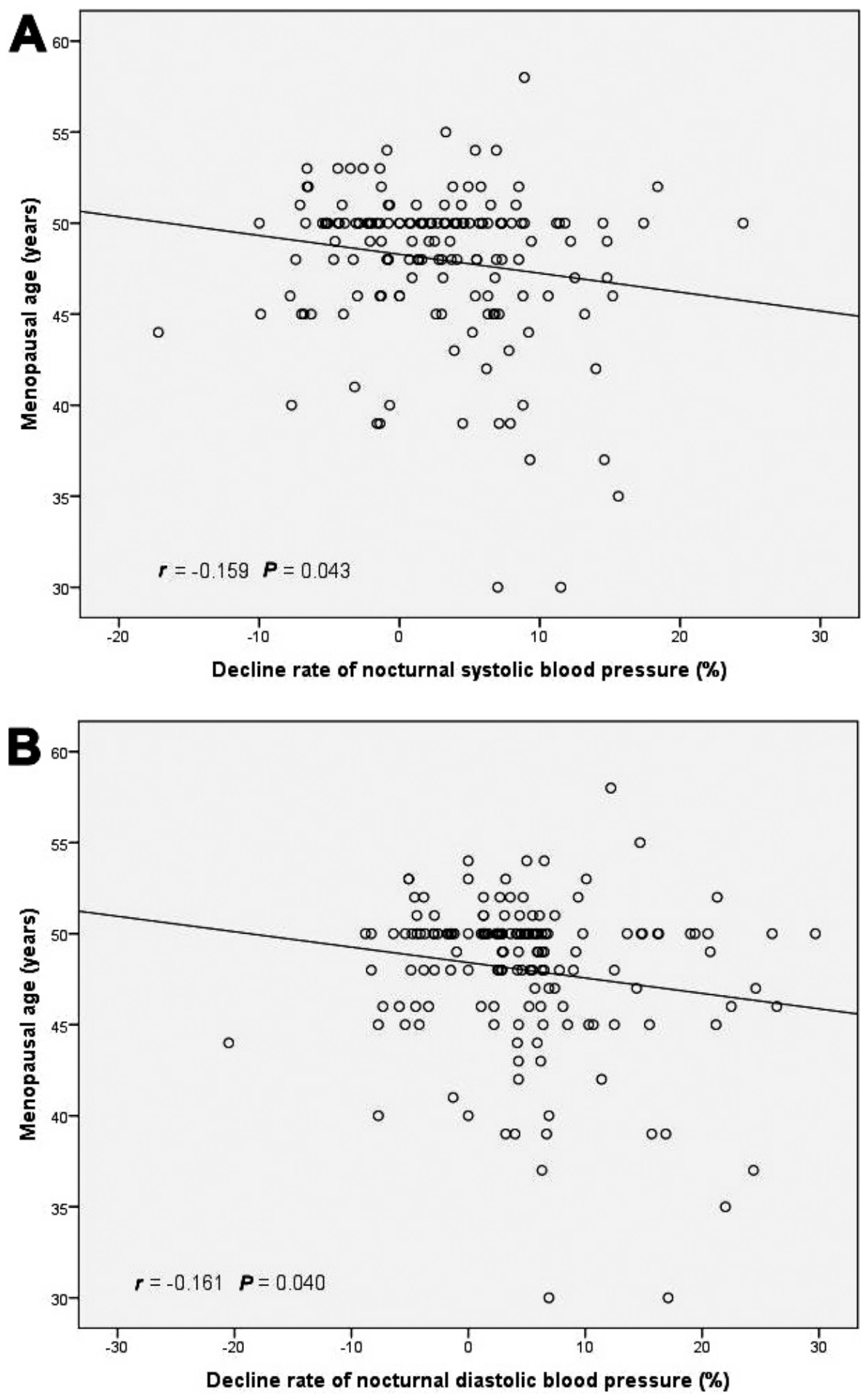

Figure 2. A: The relationship of menopause age and decline rate of nocturnal SBP. B: The relationship of menopause age and decline rate of nocturnal DBP.

was found to be predictor of cardiovascular events in hypertensive patients. ${ }^{24}$ We have also previously found that reverse-dipper pattern of BP was significantly associated with the type 2 diabetes, carotid atherosclerosis and lacuna infarction. ${ }^{11-13)}$ Hence, restoration of the physiological circadian rhythm may be an aspect of crucial importance in the treatment of hypertensive patients independent of normalization of elevated BP values. ${ }^{25}$

Menopause is the permanent cessation of menstruation, which is characterized by physiological changes that 
influence several organs and systems. The lower estrogen level of postmenopausal women is generally regarded as the main reason for an increased risk of cardiovascular diseases. ${ }^{4}$ Studies have shown that women are at a lesser risk of developing cardiovascular disease than their male counterparts before menopause, but this advantage is abolished after menopause. ${ }^{26-28)}$ Additionally, the Framingham Study suggested that female CHD morbidity rates accelerate more quickly than do those of males after the age of 45 years. $^{29)}$ So, identification of women with early menopause offers a window of opportunity to implement interventions that will improve overall cardiovascular health during the postmenopausal years.

Despite the extensive research on the relationship of menopause age and cardiovascular disease, it is not yet clear whether menopause age associated with circadian variations of $\mathrm{BP}$, in which reverse-dipper has be demonstrated as a risk of CHD in essential hypertension patients in our previous study (unpublished data). To our knowledge, this is the first study to report the relationship between menopause age and circadian blood pressure variations. We found that menopause age of reverse-dipper BP pattern was significantly later than dipper pattern. Furthermore, the menopause age was negatively correlated with the decline rate of nocturnal SBP and DBP. Our study suggested that the menopause age might serve as an independent risk factor for reverse-dipper BP pattern in postmenopausal females with hypertension. The possible mechanism of the relationship between menopause age and circadian variations of BP is proposed to be estrogen's deficiency inducing endothelial dysfunction, dyslipidemia and increased body mass index in postmenopausal $^{30)}$ However, the detailed mechanism remains to be further investigated.

Study limitations: Several potential limitations of our study should be noted. First, our study had a crosssectional design; it is possible that the results might be affected by the difference of duration between the time of 24-hour ABPM and the time of menopause among each group of circadian blood pressure, whereas a longer period of prospective observation may provide more prognostic information. Secondly, it will be more powerful if a larger sample size is employed. Thirdly, the reproducibility of ABPM is controversial because of the large influence of daily activity on BP. ${ }^{31)}$ Finally, the patients in our study are exclusively limited to northern Chinese patients from a single center, so the results should not be extended to different ethnic groups. Therefore, further prospective clinical observation with more rigorous design is necessary.

\section{Disclosures}

Conflicts of interest: The authors have no conflicts of interest regarding the content of the manuscript.

\section{References}

1. Thom T, Haase N, Rosamond W, et al. Heart disease and stroke statistics--2006 update: a report from the American Heart Asso- ciation Statistics Committee and Stroke Statistics Subcommittee. Circulation 2006; 113: e85-151.

2. Kannel WB, Hjortland MC, McNamara PM, Gordon T. Menopause and risk of cardiovascular disease: the Framingham study. Ann Intern Med 1976; 85: 447-52.

3. Muka T, Oliver-Williams C, Kunutsor S, et al. Association of age at onset of menopause and time since onset of menopause with cardiovascular outcomes, intermediate vascular traits, and all-cause mortality: a systematic review and meta-analysis. JAMA Cardiol 2016; 1: 767-76.

4. Appiah D, Schreiner PJ, Demerath EW, Loehr LR, Chang PP, Folsom AR. Association of age at menopause with incident heart failure: a prospective cohort study and meta-analysis. J Am Heart Assoc 2016; 5: e003769.

5. Burt VL, Whelton P, Roccella EJ, et al. Prevalence of hypertension in the US adult population. Results from the Third National Health and Nutrition Examination Survey, 1988-1991. Hypertension 1995; 25: 305-13.

6. Agarwal R, Andersen MJ. Prognostic importance of ambulatory blood pressure recordings in patients with chronic kidney disease. Kidney Int 2006; 69: 1175-80.

7. Bliziotis IA, Destounis A, Stergiou GS. Home versus ambulatory and office blood pressure in predicting target organ damage in hypertension: a systematic review and meta-analysis. J Hypertens 2012; 30: 1289-99.

8. Mancia G, Fagard R, Narkiewicz K, et al. ESH/ESC guidelines for the management of arterial hypertension: the Task Force for the Management of Arterial Hypertension of the European Society of Hypertension (ESH) and of the European Society of Cardiology (ESC). Eur Heart J 2013; 34: 2159-219.

9. Sarafidis PA, Rumjon A, Macdougall IC. Ambulatory blood pressure monitoring: an invaluable tool comes of age for patients with chronic kidney disease? Am J Nephrol 2012; 35: 238-41.

10. Bouhanick B, Bongard V, Amar J, Bousquel S, Chamontin B. Prognostic value of nocturnal blood pressure and reversedipping status on the occurrence of cardiovascular events in hypertensive diabetic patients. Diabetes Metab 2008; 34: 560-7.

11. Yan B, Peng L, Dong Q, et al. Reverse-dipper pattern of blood pressure may predict lacunar infarction in patients with essential hypertension. Eur J Neurol 2015; 22: 1022-5.

12. Yan B, Peng L, Han D, et al. Blood pressure reverse-dipping is associated with early formation of carotid plaque in senior hypertensive patients. Medicine 2015; 94: e604.

13. Sun L, Yan B, Gao Y, et al. Relationship between blood pressure reverse dipping and type 2 diabetes in hypertensive patients. Sci Rep 2016; 6: 25053.

14. Espinosa R, Spruill TM, Zawadzki MJ, et al. Can blood pressure measurements taken in the physician's office avoid the 'white coat' bias? Blood Press Monit 2011; 16: 231-7.

15. Verdecchia P. Prognostic value of ambulatory blood pressure: current evidence and clinical implications. Hypertension 2000; 35: 844-51.

16. O'Brien E. Dipping comes of age: the importance of nocturnal blood pressure. Hypertension 2009; 53: 446-7.

17. Verdecchia P, Porcellati C, Schillaci G, et al. Ambulatory blood pressure. An independent predictor of prognosis in essential hypertension. Hypertension 1994; 24: 793-801.

18. Ben-Dov IZ, Kark JD, Ben-Ishay D, Mekler J, Ben-Arie L, Bursztyn M. Predictors of all-cause mortality in clinical ambulatory monitoring: unique aspects of blood pressure during sleep. Hypertension 2007; 49: 1235-41.

19. Ruan Y, Wei W, Yan J, et al. Time rate of blood pressure variation is associated with endothelial function in patients with metabolic syndrome. Int Heart J 2016; 57: 226-33.

20. Cuspidi C, Meani S, Lonati L, et al. Short-term reproducibility of a non-dipping pattern in type 2 diabetic hypertensive patients. J Hypertens 2006; 24: 647-53.

21. Vasunta RL, Kesaniemi YA, Ylitalo A, Ukkola O. Nondipping pattern and carotid atherosclerosis in a middle-aged population: 
OPERA Study. Am J Hypertens 2012; 25: 60-6.

22. Ibernon M, Moreso F, Sarrias X, et al. Reverse dipper pattern of blood pressure at 3 months is associated with inflammation and outcome after renal transplantation. Nephrol Dial Transplant 2012; 27: 2089-95.

23. Wang C, Zhang J, Liu X, et al. Reversed dipper blood-pressure pattern is closely related to severe renal and cardiovascular damage in patients with chronic kidney disease. PloS one 2013; 8: e55419.

24. Kario K, Pickering TG, Matsuo T, Hoshide S, Schwartz JE, Shimada K. Stroke prognosis and abnormal nocturnal blood pressure falls in older hypertensives. Hypertension 2001; 38: 852-7.

25. Schillaci G, Battista F, Settimi L, Schillaci L, Pucci G. Antihypertensive drug treatment and circadian blood pressure rhythm: a review of the role of chronotherapy in hypertension. Curr Pharm Des 2015; 21: 756-72.

26. Reddy Kilim S, Chandala SR. A comparative study of lipid pro- file and oestradiol in pre- and post-menopausal women. J Clin Diagn Res 2013; 7: 1596-8.

27. Ratiani L, Khorava M, Dgebuadze M, Zhvania N, Sanikidze T. The role of estrogens in pathogenesis of age-related arterial hypertension. Georgian Med New 2012; 71-6.

28. Yihua L, Yun J, Dongshen Z. Coronary Artery Disease in Premenopausal and Postmenopausal Women. Int Heart J 2017; 58: 174-9.

29. Lerner DJ, Kannel WB. Patterns of coronary heart disease morbidity and mortality in the sexes: a 26-year follow-up of the Framingham population. J Am Heart 1986; 111: 383-90.

30. Modena MG. Hypertension in postmenopausal women: how to approach hypertension in menopause. High Blood Press Cardiovasc Prev 2014; 21: 201-4.

31. Dimsdale JE, von Kanel R, Profant J, Nelesen R, Ancoli-Israel S, Ziegler M. Reliability of nocturnal blood pressure dipping. Blood Press Monit 2000; 5: 217-21. 